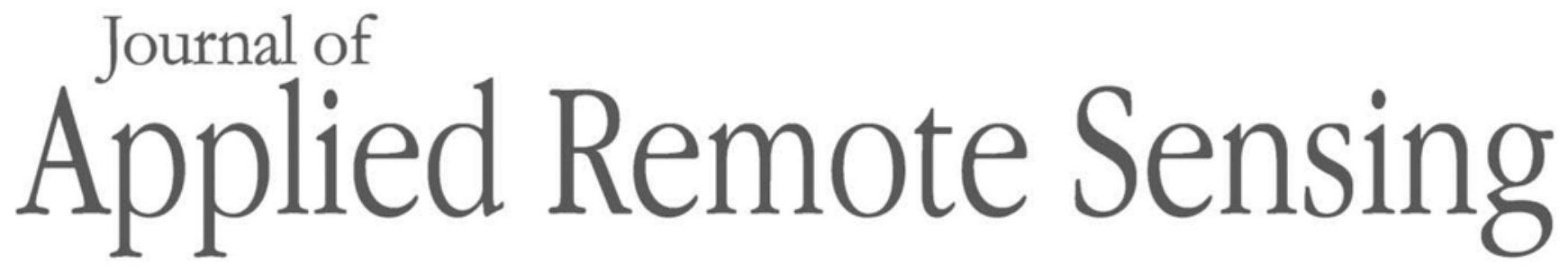

RemoteSensing.SPIEDigitalLibrary.org

\title{
Parameter estimation and imaging of moving targets in bistatic synthetic aperture radar
}

\author{
$\mathrm{Yu} \mathrm{Li}$ \\ Puming Huang \\ Zhimei Yang \\ Chenchen Lin
}




\title{
Parameter estimation and imaging of moving targets in bistatic synthetic aperture radar
}

\author{
Yu Li,* Puming Huang, Zhimei Yang, and Chenchen Lin \\ Xi'an Institute of Space Radio Technology, No. 150, Weiqu Street, \\ Changan District, Xi' an 710100, China
}

\begin{abstract}
In high-resolution bistatic synthetic aperture radar (SAR) systems, parameter estimation is essential to moving target imaging quality. However, precise parameters are difficult to obtain without priori information due to the relative along-track and across-track velocities between the moving target and platforms that change with time. A parameter estimation and imaging approach for moving targets is proposed. First, slant range and relative velocities expression are deduced based on the geometry of bistatic SAR model with one stationary configuration. Then, range curvature term are compensated skillfully by fitting the range-compressed curve in two-dimensional time domain, meanwhile, the initial estimated range walk slope can be achieved. Finally, precise Doppler centroid is estimated through searching for the maximum contrast with folding search algorithm, which is giving consideration to both searching precision and computational complexity. Thus, the proposed algorithm provides an effective way for parameter estimation and imaging of moving target without prior information and interpolation operation. Experimental results show the effectiveness of the proposed method. () The Authors. Published by SPIE under a Creative Commons Attribution 3.0 Unported License. Distribution or reproduction of this work in whole or in part requires full attribution of the original publication, including its DOI. [DOI: 10.1117/1.JRS.10.015018]
\end{abstract}

Keywords: bistatic synthetic aperture radar; parameter estimation; curve fitting; maximum contrast; folding search algorithm.

Paper 15669 received Sep. 23, 2015; accepted for publication Feb. 23, 2016; published online Mar. 16, 2016.

\section{Introduction}

In bistatic SAR data acquisition process, beam center offset would reduce signal-to-noise ratio, increase main lobe width and change focusing position, which are caused by motion error of platforms and beam pointing error of antennas. Therefore, accurate Doppler centroid estimation is the premise of high-quality moving target imaging. In general, the Doppler centroid estimation processing includes the base-band Doppler centroid estimation and the Doppler ambiguity number assessing, in which a minor error range is allowed when estimating the base-band Doppler centroid, while the Doppler ambiguity number is required to be completely consistent with the theoretical value.

Presently, several Doppler centroid estimation techniques have been proposed to deal with the Doppler ambiguity problem. By applying an average cross correlation coefficient (ACCC) to signals in range frequency domain, wavelength diversity algorithm (WDA) ${ }^{1}$ obtains absolute Doppler centroid according to the relation between Doppler centroid and range frequency. Multilook cross correlation (MLCC) ${ }^{2}$ and multilook beat frequency (MLBF) $)^{3}$ resolve Doppler ambiguity through decomposing the original signal into two subsignals with different carrier frequencies. MLCC achieves Doppler centroid by calculating the phase difference of ACCC between subsignals, while the latter method gets that directly via computing the frequency difference of subsignals. Among the mentioned three algorithms, WDA and MLCC are suitable for low-contrast scene, and MLBF is propitious to high-contrast scene. Multiple PRF $^{4-6}$ avoids Doppler ambiguity based on a set of PRFs, whose disadvantages lie in the complexity of system design. Therefore, this method is employed mostly in ScanSAR mode.

*Address all correspondence to: Yu Li, E-mail: liy_504china@163.com 
Radon transform ${ }^{7-9}$ has been proved to be a high-precision method to estimate Doppler centroid in medium and high-contrast scenes, which give accurate Doppler centroid estimation based on the linear feature of the range-compressed signal. However, the estimation accuracy deteriorates when the range curvature cannot be neglected. Moreover, it is confronted with great computational complexity. In this paper, a new parameter estimation and imaging approach for moving targets is proposed. First, the expression of slant range is derived and approximated based on the bistatic SAR model, from which we know the along-track and across-track velocity cannot be solved without prior information. Then, curve fitting is applied to range-compressed signal in two-dimensional (2-D) time domain. On this basis, precise Doppler center is estimated using high-efficient folding search algorithm. The proposed algorithm does not involve interpolation operation and provides an effective way for parameter estimation and imaging in bistatic SAR.

\section{Bistatic Synthetic Aperture Radar with One Stationary Configuration}

\subsection{Geometry Model}

The geometry model of bistatic SAR is shown in Fig. 1. Assume that the transmitter is fixed, and the receiver moves along the $x$-axis direction and works at side looking mode with velocity $V_{R}$. $R_{T 0}$, and $R_{R 0}$ are the slant range from the transmitter and receiver to ground moving target at $t_{a}=0$, while $R_{T 1}$ and $R_{R 1}$ stand for the instantaneous slant ranges of the transmitter and receiver from the target. $\theta_{T}$ and $\theta_{R}$ are the squint angles from the two platforms to the target, $t_{a}$ is the slow time. $v_{T a}, v_{R a}, v_{T r}$, and $v_{R r}$ denote the relative lateral and radial velocities between the moving target and platforms, respectively. The velocity of the moving target $v$ can be decomposed into $v_{T a}$ and $\widetilde{v_{T \mathrm{r}}}$. Thus, the instantaneous slant range $R\left(t_{a}\right)$ is given by

$$
\begin{aligned}
R\left(t_{a}\right)= & \sqrt{\left(R_{T 0}-v_{T r} t_{a}\right)^{2}+\left(v_{T a} t_{a}\right)^{2}+2\left(R_{T 0}-v_{T r} t_{a}\right)\left(v_{T a} t_{a}\right) \sin \theta_{T}} \\
& +\sqrt{\left(R_{R 0}-v_{R r} t_{a}\right)^{2}+\left(V_{R} t_{a}-v_{R a} t_{a}\right)^{2}} .
\end{aligned}
$$

According to the geometry of bistatic SAR model with one stationary configuration, we have

$$
v / \sin \left(\pi / 2-\theta_{T 1}\right)=v_{T a} / \sin \left(\pi / 2+\theta_{T 1}-\theta_{1}\right)=\widetilde{v_{T \mathrm{r}}} / \sin \theta_{1} .
$$

Thus, $v_{T a}, v_{R a}, v_{T r}$, and $v_{R r}$ is given by

$$
\left\{\begin{array}{l}
v_{T r}=\widetilde{v_{T \mathrm{r}}} \cos \varepsilon_{T}=v \sin \theta_{1} \cos \varepsilon_{T} / \sin \left(\pi / 2-\theta_{T 1}\right) \\
v_{T a}=v \sin \left(\pi / 2+\theta_{T 1}-\theta_{1}\right) / \sin \left(\pi / 2-\theta_{T 1}\right)
\end{array} .\right.
$$

Similarly, we have

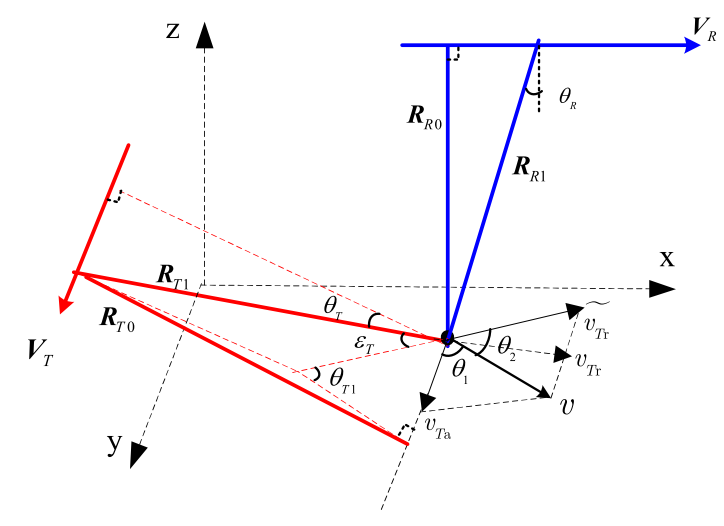

Fig. 1 Geometry model. 


$$
\left\{\begin{array}{l}
v_{R r}=v \cos \theta_{1} \cos \varepsilon_{R} \\
v_{R a}=v \sin \theta_{1}
\end{array}\right.
$$

where $\theta_{1}$ and $\theta_{2}$ represent the angles between $v_{T a}, v_{R a}$, and $v$, respectively. $\varepsilon_{T}$ and $\varepsilon_{R}$ are the grazing angles of transmitter and receiver. Obviously, $\theta_{1}, \theta_{2}$, and $\theta_{T 1}$ satisfies

$$
\left\{\begin{array}{l}
\theta_{1}+\theta_{2}+\left(\pi / 2-\theta_{T 1}\right)=\pi \\
\theta_{T 1}=\sin ^{-1}\left(\sin \theta_{T} / \cos \varepsilon_{T}\right)
\end{array} .\right.
$$

From Eqs. (2)-(4), one obtains six equations with eight variables, i.e., $v_{T a}, v_{R a}, v_{T r}, v_{R r}, \theta_{1}, \theta_{2}$, $\theta_{T 1}$, and $v$. In order to calculate these variables, range walk and range curvature term are estimated in the following procedures, both of which are the functions of the unknown parameters. In this way, the moving target-related variables can be achieved.

\subsection{Analysis of Range Migration}

Ignoring higher-order terms of phase, the Tailor series expansion coefficients of $R\left(t_{a}\right)$ at the beam center crossing time is given by ${ }^{10-11}$

$$
R\left(t_{a}\right) \approx R_{T 0}+R_{R 0}-v_{T r} t_{a}+v_{T a} t_{a} \sin \theta_{T}-v_{R r} t_{a}+\frac{\left(v_{T a} t_{a} \cos \theta_{T}\right)^{2}}{2 R_{T 0}}+\frac{\left(V_{R} t_{a}-v_{R a} t_{a}\right)^{2}}{2 R_{R 0}} .
$$

According to the analysis of range migration given in Ref. 12, the Doppler centroid can be easily deduced when the range walk is far greater than the range curvature, and the quadratic term in Eq. (5) can be neglected. That is

$$
\left|v_{T r} t_{a}+v_{R r} t_{a}-v_{T a} t_{a} \sin \theta_{T}\right| \gg\left|\frac{\left(v_{T a} t_{a} \cos \theta_{T}\right)^{2}}{2 R_{T 0}}+\frac{\left(V_{R} t_{a}-v_{R a} t_{a}\right)^{2}}{2 R_{R 0}}\right| .
$$

However, the above assumption would no longer hold in some cases, e.g., the platform height is low, the beam width is broad, and the receiving platform velocity is high. Thus, the Doppler centroid cannot be estimated accurately using the algorithm in Ref. 12 .

\section{Proposed Parameter Estimation Algorithm}

In this section, at first we introduce curve fitting method to achieve the linear and quadratic coefficients of bistatic slant range. Then precise Doppler centroid can be obtained effectively through contrast-based folding search algorithm. Finally, moving target can be well focused using the estimated parameters.

\subsection{Curve Fitting Method}

Suppose a linear FM signal is transmitted, then the received signal after range compression is given by

$s\left(f_{r}, t_{a}\right)=w_{a}\left(t_{a}\right) w_{r}\left(f_{r}\right) \exp \left\{-j \frac{2 \pi\left(f_{0}+f_{r}\right)}{c}\left[\begin{array}{l}R_{T 0}+R_{R 0}-v_{T r} t_{a}-v_{R r} t_{a}+v_{T a} t_{a} \sin \theta_{T} \\ +\frac{\left(v_{T a} t_{a} \cos \theta_{T}\right)^{2}}{2 R_{T 0}}+\frac{\left(V_{R} t_{a}-v_{R a} t_{a}\right)^{2}}{2 R_{R 0}}\end{array}\right]\right\}$,

where $w_{a}(\cdot)$ and $w_{r}(\cdot)$ are the range and azimuth envelopes, respectively.

Due to the separated transmitting and receiving antennas, relative along-track and acrosstrack velocities between the moving target and platforms change with time, which lead to the linear and quadratic terms of $t_{a}$ in slant range, as is indicated in Eq. (6). Obviously, range curvature compensation is the premise of Doppler centroid estimation, but the relative velocities are unknown and cannot be obtained directly from the echo data with bistatic configuration. Therefore, we could not get range curvature compensation factor without priori 
information. Accordingly, the existent of quadratic term makes the Doppler centroid estimation method $^{12}$ not applicable.

In this paper, considering the bending property of the signal in 2-D time domain, the bending degree stands for the quadratic coefficient, range curvature term is compensated by curve fitting method. Meanwhile, the linear coefficient is used for the rough estimation of Doppler centroid. In order to improve the fitting accuracy, the curve is refined before fitting operation. Assuming that the linear and quadratic coefficients achieved by curve fitting method are $T_{1}$ and $T_{2}$, respectively, where $T_{1}$ denotes the Doppler centroid offset and $T_{2}$ represents the defocusing degree. In the process of parameter estimation, range curvature term is first compensated, and then range walk slope is estimated on the basis of this procedure. Theoretically, $T_{1}=-\left.\left[d R\left(t_{a}\right) / d t_{a}\right]\right|_{t_{a}=0}$, $T_{2}=-\left.\left[d^{2} R\left(t_{a}\right) / d t_{a}^{2}\right]\right|_{t_{a}=0}$. Range curvature compensation factor is given by

$$
H_{1}\left(f_{r}, t_{a}\right)=\exp \left[j \frac{2 \pi}{c}\left(f_{c}+f_{r}\right) T_{2} t_{a}^{2}\right] .
$$

\subsection{Definition of Contrast}

Assume that the range curvature term has been compensated, then the relationship among the range walk term, the slope $K$ and the Doppler centroid $f_{d c}$ can be written as

$$
f_{d c}=-K / \lambda=\left(v_{T r}+v_{R r}-v_{T a} \sin \theta_{T}\right) / \lambda \approx-T_{1} / \lambda .
$$

Initial Doppler centroid correction factor is given by

$$
H_{2}=\exp \left[j \frac{2 \pi\left(f_{r}+f_{c}\right)}{c} T_{1} t_{a}\right] .
$$

Here, we introduce the concept of contrast. ${ }^{13,14}$

Parameter estimation method based on the contrast possesses better performance than that based on the variance or Radon transform,${ }^{14}$ which has been widely used in the field of SAR imaging. Assuming that the resolution cell in range direction is $N_{r}, x(n)\left(n=0,1,2, \ldots, N_{r}-\right.$ $1)$ indicates the accumulated energy along azimuth direction after Doppler centroid correction. From Eq. (6), we know the target velocity $v$ and the squint angle $\theta_{T}$ are variables in $x(n)$. Thus, definition of contrast can be expressed as

$$
C\left(v, \theta_{T}\right)=\frac{\sqrt{\frac{1}{N_{r}} \sum_{n=0}^{n=N_{r}-1}\left[I^{2}(n)-\frac{1}{N_{r}} \sum_{n=0}^{n=N_{r}-1} I^{2}(n)\right]^{2}}}{\frac{1}{N_{r}} \sum_{n=0}^{n=N_{r}-1} I^{2}(n)},
$$

where $I(n)$ is defined as

$$
I(n)=\frac{|x(n)|}{\|x\|}, \quad\|x\|=\sum_{n=0}^{N_{r}-1}|x(n)| .
$$

From the above definition, we know contrast reflects the energy accumulation degree. That is, if the contrast is large, the accumulated energy along azimuth direction gathers near one range cell. Otherwise, small contrast means the energy gathers in several or more range cells. In this case, defocusing phenomena will appear after range compression. As a result, the exact Doppler centroid estimation is equivalent to searching the maximum contrast.

\subsection{Folding Search Algorithm}

The conventional ways for searching for range walk slope are based on one fixed step. These methods may result in many shortcomings. On the one hand, supposing that the initial estimated slope deviation has the same sign as the searching step, the estimated slope error will increase with the searching process, and the optimal slope cannot be obtained. On the other hand, when 
the initial slope error is large or the required Doppler centroid accuracy is high, conventional searching methods will face huge computational complexity. In view of the above problems, a folding search algorithm based on maximum contrast is presented in this section, the main steps are as follows:

- Step 1: Initializing step $\Delta K$ and setting $\Delta K_{0}$ as the termination step in folding search algorithm.

- Step 2: Equalizing range curvature term using the curve fitting method.

- Step 3: Initializing range walk compensation factor with the fitting coefficient, and calculating the initial contrast $C_{1}$ according to Eq. (10).

- Step 4: Updating the contrast $C_{k}(k=2,3,4 \ldots)$. If $C_{k} \geq C_{k-1}$, continue this iteration process. Otherwise, turn to step 5.

- Step 5: Outputting the slope and corresponding Doppler centroid, if the step is less than $\Delta K_{0}$.

Analysis of computational complexity: Assuming that the initial step error is $\Delta E$, the computational complexity of folding search algorithm based on maximum contrast is $\log _{2}\left(\Delta E / \Delta K_{0}\right)$, while the conventional algorithm based on fixed step is $\Delta E / \Delta K_{0}$. With the increase of initial step error and enhancement of step accuracy, the computational complexity of the proposed algorithm is significantly reduced compared to that of conventional algorithms.

\subsection{Moving Target Imaging Procedure}

At this stage, moving target can be imaged by means of the estimated linear and quadratic coefficients of bistatic slant range. Assuming that the ultimate range walk slope using folding search algorithm is $T_{3}$, then the final Doppler centroid correction factor is given by

$$
H_{3}\left(f_{r}, t_{a}\right)=\exp \left[j \frac{2 \pi\left(f_{r}+f_{c}\right)}{c} T_{3} t_{a}\right] .
$$

Multiplying Eq. (6) with Eq. (11), and transforming the results into 2-D frequency domain yields

$$
S\left(f_{r}, f_{a}\right)=\exp \left(-j\left\{\frac{2 \pi\left(f_{r}+f_{c}\right)}{c}\left(R_{T 0}+R_{R 0}\right)-\frac{\pi c f_{a}^{2}}{2\left(f_{r}+f_{c}\right)\left[\frac{\left(v_{T a} t_{a} \cos \theta_{T}\right)^{2}}{2 R_{T 0}}+\frac{\left(V_{R} t_{a}-v_{R a} t_{a}\right)^{2}}{2 R_{R 0}}\right]}\right\} .\right.
$$

In Eq. (12), as the linear term of $t_{a}$ mainly affects the envelope position, so it cannot be reflected in phase term. Finally, the azimuth compression factor is given by

$$
H_{4}\left(f_{r}, f_{a}\right)=\exp \left[-j \frac{\pi c f_{a}^{2}}{2\left(f_{r}+f_{c}\right) T_{2}}\right] .
$$

After transforming the azimuth-compressed signal into 2-D time domain, moving target imaging process with bistatic configuration is accomplished. The final imaging quality depends on the accuracy of fitting coefficients as well as the termination step in folding search algorithm. Figure 2 shows the flow chart of the proposed parameter estimation algorithm.

\section{Simulation Results}

In this section, simulation results of different algorithms are compared to evaluate the effectiveness of the proposed one. Assume four moving targets with the same reflectivity coefficients are located in the scene. The positions of target 1 , target 2 , target 3 , and target 4 are given by $(0,400)$ $\mathrm{m},(0,-800) \mathrm{m},(62.5,0) \mathrm{m}$, and $(-375,0) \mathrm{m}$, respectively. Among the moving targets, target 1 and target 4 have the same velocity $15 \mathrm{~m} / \mathrm{s}$ and the same angle 12 deg between target velocity 
Li et al.: Parameter estimation and imaging of moving targets in bistatic synthetic aperture radar

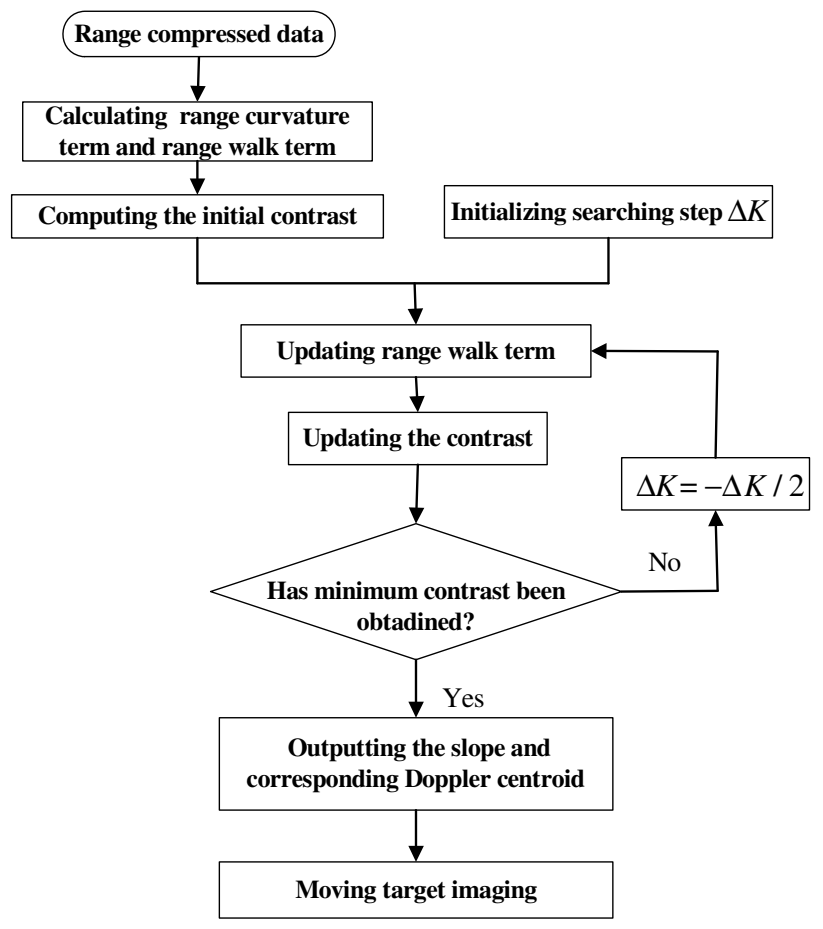

Fig. 2 Flow chart of the proposed algorithm.

and $y$-axis, while target 2 and target 3 have the same velocity $17 \mathrm{~m} / \mathrm{s}$ and the same angle $10 \mathrm{deg}$ between target velocity and $y$-axis. Other simulation parameters are shown in Table 1 .

\subsection{Simulations for Multiple Moving Targets}

In Sec. 3, we apply curve fitting method to the range-compressed signal. In this way, the coefficients that represent the initial range walk slope and range curvature term are obtained. Figure 3(a) shows the range-compressed signal in 2-D time domain. Figure 3(b) denotes the initial range walk slope corrected signal, which indicate that different targets energy are gathered

Table 1 Simulation parameters.

\begin{tabular}{lc}
\hline \hline Parameter & Value \\
\hline Coordinates of transmitter & $(-3,-2,1) \mathrm{km}$ \\
Coordinates of receiver & $(-15,0,1) \mathrm{km}$ \\
Bandwidth & $95 \mathrm{MHz}$ \\
Beam width of transmitter & $10 \mathrm{deg}$ \\
Carrier frequency & $10 \mathrm{GHz}$ \\
PRF & $400 \mathrm{~Hz}$ \\
Synthetic aperture time & 10.50 \\
Receiver velocity & $100 \mathrm{~m} / \mathrm{s}$ \\
Initial searching precision & $0.2 \mathrm{~m} / \mathrm{s}$ \\
Beam width of receiver & $4 \mathrm{deg}$ \\
Terminal searching precision & $0.02 \mathrm{~m} / \mathrm{s}$ \\
\hline \hline
\end{tabular}



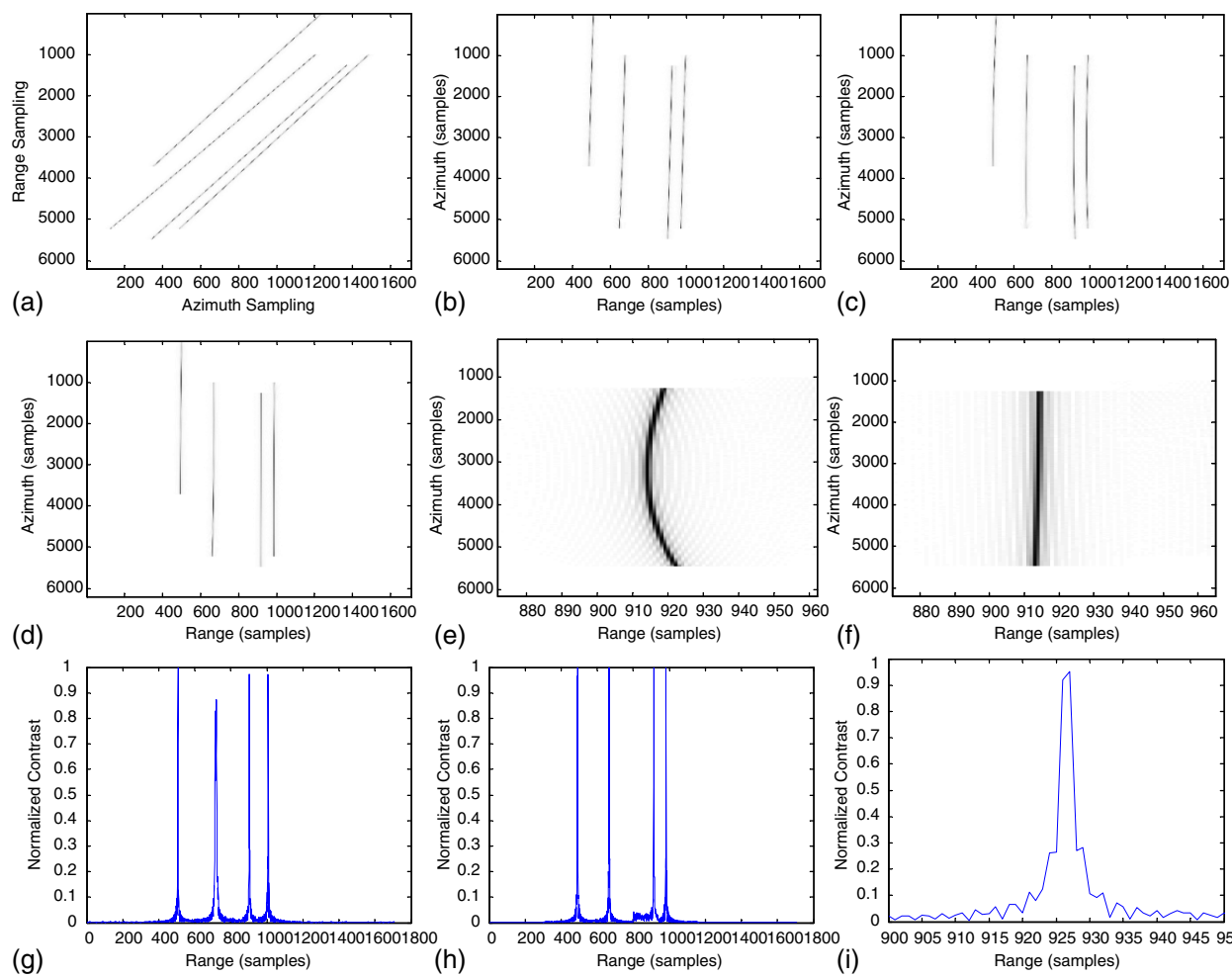

(e)
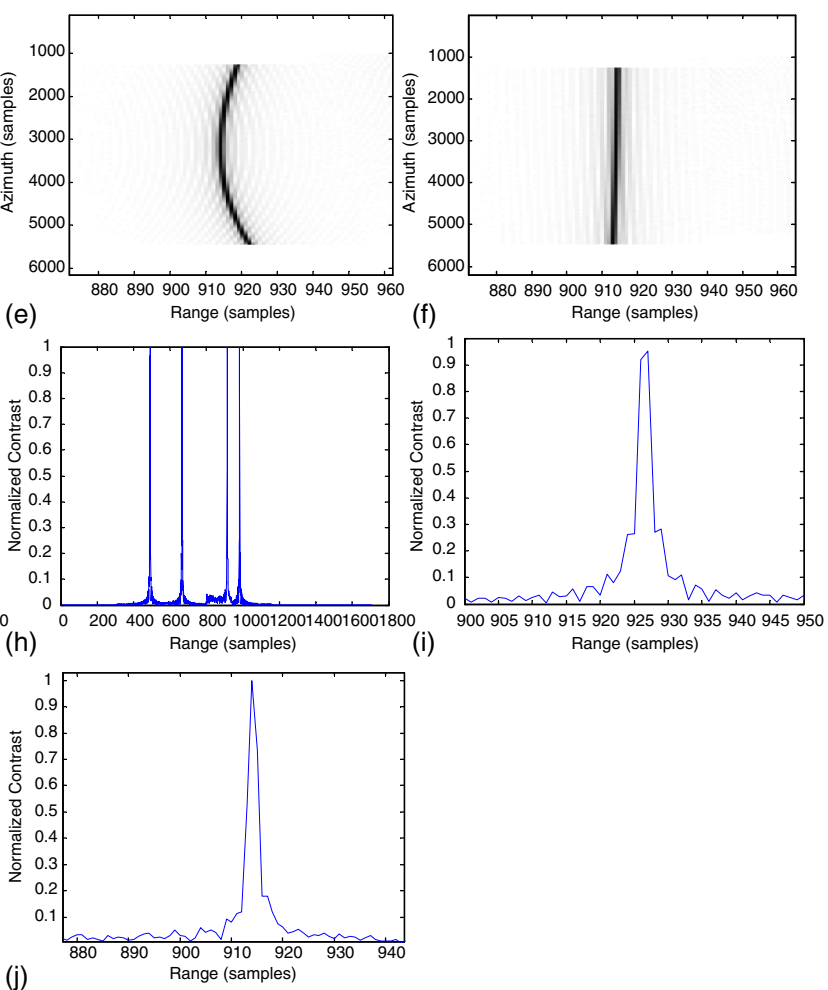

Range (samples)

Fig. 3 Simulations of multiple moving targets: (a) range-compressed data, (b) initial range walk slope corrected data, (c) range corrected data using paper ${ }^{12}$ algorithm, (d) range corrected data using the proposed algorithm, (e) target 3 without range curvature correction, (f) target 3 after range curvature correction, (g) energy accumulation results using paper ${ }^{12}$ algorithm, (h) energy accumulation results using the proposed algorithm, (i) amplified target 3 from (g), and (j) amplified target 3 from (h).

into different range intervals. As a result, folding search algorithm can be employed to conduct energy accumulation for multiple targets. Here, the slope $K$ of the four targets in Fig. 3(b) are $-177,-179.5,-183$, and -191 . Figures 3(c) and 3(d) are the range corrected signal using paper $^{12}$ algorithm and the proposed algorithm, respectively. In order to evaluate the effectiveness of our method, Figs. 3(e) and 3(f) give the amplified curve of target 3, in which the curve in Fig. 3(e) becomes a straight line, as is shown in Fig. 3(f). This phenomenon results from the fact that the range curvature term has been compensated with the proposed method. Figures $3(\mathrm{~g})$, 3(h), 3(i), and 3(j) show the comparison of the normalized contrast between the folding search algorithm and paper ${ }^{12}$ algorithm. From the comparison between Figs. 3(i) and 3(j), we know the normalized contrast in Fig. 3(i) focuses on a finite range interval because the range curvature term is ignored with paper ${ }^{12}$ algorithm, while the proposed algorithm avoids this problem, and the energy gathers more intensively. Obviously, the latter is more beneficial for high-accuracy Doppler centroid estimation.

The estimated Doppler centroid error and computing time using different algorithms are listed in Table 2. By adopting curve fitting method and high-efficient search algorithm, not only the performance but the computation complexity is taken into account in the proposed algorithm even though insufficient prior information is provided, which is consistent with 
Table 2 Comparisons of estimation results.

\begin{tabular}{lccc}
\hline \hline & $\begin{array}{c}\text { Doppler centroid errors of target 1, Velocity errors of target 1, target 2, } \\
\text { target 2, target 3, and target 4 }(\mathrm{Hz})\end{array}$ & $\begin{array}{c}\text { Computation } \\
\text { target 3, and target 4 }(\mathrm{m} / \mathrm{s})\end{array}$ \\
\hline Radon transform & $3.60,7.05,4.34,6.88$ & $0.5040,1.2690,0.7812,0.9632$ & 120.80 \\
Paper & & & \\
Proposed method & $2.48,4.31,2.82,4.46$ & $0.3472,0.7758,0.5076,0.6244$ & 61.33 \\
Propod & $0.46,0.71,0.42,0.67$ & $0.0644,0.1278,0.0756,0.0938$ & 28.01 \\
\hline \hline
\end{tabular}

the theoretical analysis. On the other hand, velocity errors of different targets can be achieved simultaneously from the derivation in Sec. 2. Parameter estimation error with the proposed algorithm is mainly caused by the approximation of double square-root term equation and the deficiency of compensation factor precision.

\subsection{The Influence of Parameter Error on Estimation Results}

According to the definition of contrast, it is influenced by range walk slope and range curvature term. From Eq. (10), we know these two parameters are composed of the target velocity and the transmitting angle essentially. As a result, the target velocity and the transmitting angle are the direct factor to imaging quality. Based on the parameters of target 3 , the errors of linear and quadratic coefficient caused by incorrect target velocity as well as inaccurate transmitting angle are shown in Figs. 4(a) and 4(b), respectively. It is obvious that the range walk slope is more sensitive to the parameter error compared with range curvature term. Therefore, range curvature term can be estimated simply using curve fitting method, while the assessment of range walk slope requires not only curve fitting method but the secondary estimation based on folding search algorithm.

\subsection{Comparison of Imaging Results}

In general, the final purpose of parameter estimation algorithms is to detect and image moving targets. Here, the parameters of target 3 are still employed. Comparison of imaging results using different algorithms is shown in Fig. 5, among which (a), (b), and (c) are obtained by radon transform, paper ${ }^{12}$ algorithm, and the proposed algorithm, respectively. The range interval and step size in radon transform are set to $20 \mathrm{deg}$ and $0.02 \mathrm{deg}$. Since the previous two algorithms do not give consideration to the influence of range curvature term, precise azimuth compression factor cannot be achieved. Also, defocusing phenomenon will appear after azimuth compression. ${ }^{15}$ Suppose that the azimuth compression function with a certain error is given by

$$
H_{4}\left(f_{r}, f_{a}\right)=\exp \left[-j \frac{\delta \pi c f_{a}^{2}}{2\left(f_{r}+f_{c}\right) T_{2}}\right]
$$

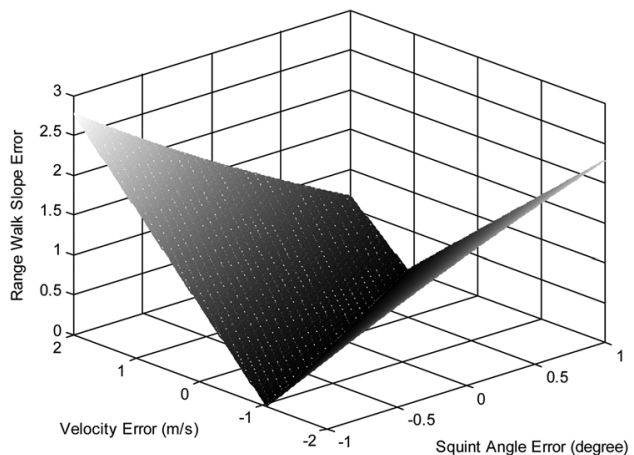

(a)

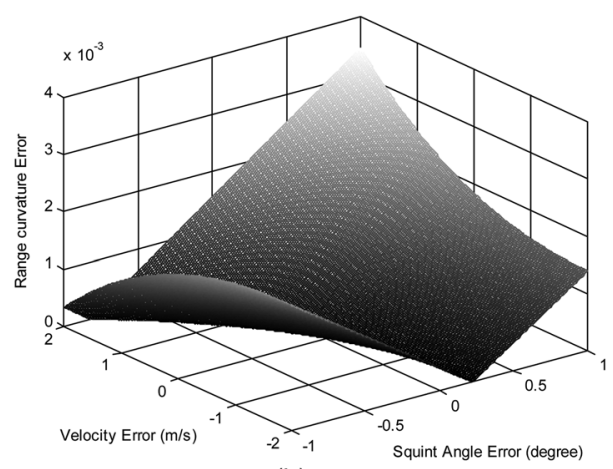

(b)

Fig. 4 Simulation results of estimation error caused by velocity and squint angle: (a) range walk slope error and (b) range curvature error. 

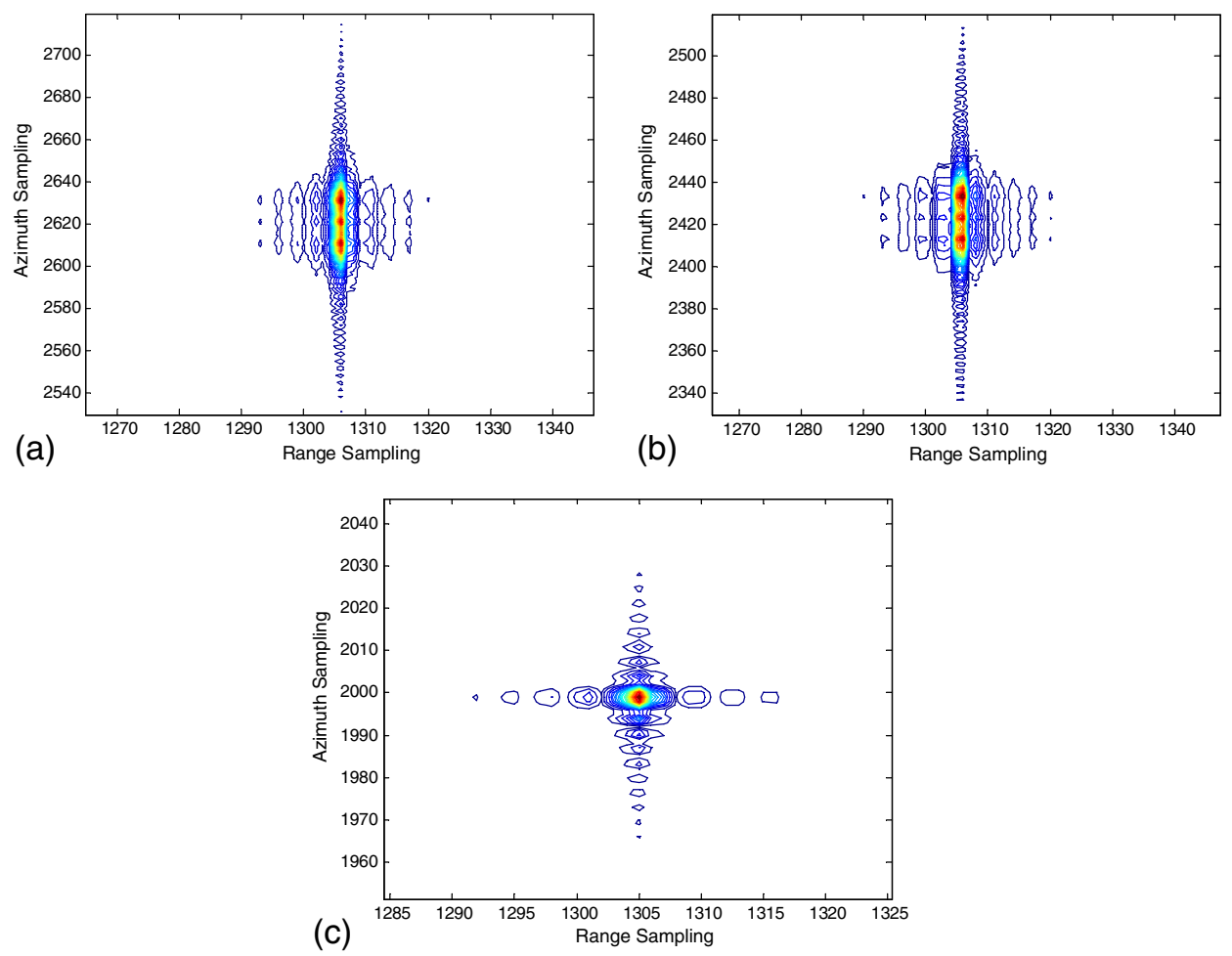

Fig. 5 Comparison of imaging results using different algorithms: (a) radon algorithm, (b) paper ${ }^{12}$ algorithm, and (c) proposed algorithm.

where the defocusing factor $\delta(0 \leq \delta \leq 1)$ is set to 0.98 . With the increase of $\delta$, azimuth focusing performance will be improved. It can be seen from Fig. 5 that even though $\delta$ is as high as 0.98 , desired imaging result cannot be achieved. Compared with the previous two algorithms, the focusing accuracy has been significantly improved using the proposed algorithm. The simulation results in this section suggest that the range curvature term is essential when the platform height is low, the beam width is broad, or the receiving platform velocity is high, which are in agreement with the theoretical analysis.

\section{Conclusion}

For moving targets imaging procedure with bistatic SAR configuration, the relative along-track and across-track velocities between the moving target and platforms are required. Therefore, four independent equations need to be constructed in theory. However, we could not achieve enough related information from the echo data without prior knowledge. In this paper, a parameter estimation and imaging algorithm for moving targets is presented. On the one hand, the expression of slant range and relative velocities are derived based on the geometry of bistatic SAR model with one stationary configuration. On the other hand, range curvature term is compensated with polynomial fitting method, and the precise Doppler centroid is obtained using folding search algorithm. Experimental results show that the proposed algorithm could give consideration to both the performance and computational complexity. In addition, this algorithm can also be employed in bistatic SAR system with arbitrary configuration.

\section{References}

1. R. Bamler and H. Runge, "PRF-ambiguity resolving by wavelength diversity," IEEE Trans. Geosci. Remote Sens. 29(6), 997-1003 (1991).

2. F. H. Wong and I. G. Cumming, "A combined SAR Doppler centroid estimation scheme based upon signal phase," IEEE Trans. Geosci. Remote Sens. 34(3), 696-707 (1996). 
3. I. G. Cumming and S. Li, "Adding Sensitivity to the MLBF Doppler centroid estimator," IEEE Trans. Geosci. Remote Sens. 45(2), 279-291 (2007).

4. C. Y. Chang and J. C Curlander, "Application of the multiple PRF technique to resolve Doppler centroid estimation ambiguity for spaceborne SAR," IEEE Trans. Geosci. Remote Sens. 30(5), 941-949 (1992).

5. M. Y. Jin, "Optimal range and Doppler centroid estimation for a ScanSAR system," IEEE Trans. Geosci. Remote Sens. 34(2), 479-488 (1996).

6. C. Cafforio and P. Guccione, "Guarnieri A.M. Doppler centroid estimation for ScanSAR data," IEEE Trans. Geosci. Remote Sens. 42(1), 14-23 (2004).

7. Y. K. Kong, B. L. Cho, and Y. S. Kim, "Ambiguity-free Doppler centroid estimation technique for airborne SAR using the Radon transform," IEEE Trans. Geosci. Remote Sens. 43(4), 715-721 (2005).

8. W. Li et al., "An improved radon-transform-based scheme of Doppler centroid estimation for bistatic forward-looking SAR," IEEE Geosci. Remote Sens. Lett. 8(2), 379-383 (2011).

9. I. G. Cumming and $\mathrm{S}$. Li, "Improved slope estimation for SAR Doppler ambiguity resolution," IEEE Trans. Geosci. Remote Sens. 44(3), 707-718 (2006).

10. S. Zhu et al., "Ground moving targets imaging algorithm for synthetic aperture radar," IEEE Trans. Geosci. Remote Sens. 49(1), 462-477 (2011).

11. F. Zhou et al., "Approach for single channel SAR ground moving target imaging and motion parameter estimation," IET Radar Sonar Navig. 1(1), 59-66 (2007).

12. W. Li et al., "A geometry-based Doppler centroid estimator for bistatic forward-looking SAR," IEEE Geosci. Remote Sens. Lett. 9(3), 388-392 (2012).

13. F. Berizzi, M. Martorella, and B. Haywood, "A contrast maximization based technique for 2D ISAR autofocusing," Proc. Inst. Electr. Eng.-Radar, Sonar, Navig. 152(4), 253-262 (2005).

14. F. Berizzi et al., "A contrast-based algorithm for synthetic range profile motion compensation," IEEE Trans. Geosci. Remote Sens. 46(10), 3053-3062 (2008).

15. Z. Li, Z. Bao, and F. Yang, "Ground moving target detection and location based on SAR images for distributed spaceborne SAR,” Sci. China Ser. F: Inf. Sci. 48(5), 632-646 (2005).

Li Yu received his BSc degree from Northwest University in 2007, and his MSc degree from Xi'an Institute of Space Radio Technology in 2011. Currently, he is a PhD candidate at Xi'an Institute of Space Radio Technology. His main research interests include moving target detection and tracking and radar imaging.

Puming Huang received his BSc degree from Harbin Institute of Technology in 1993, his MSc degree from Xi'an Institute of Space Radio Technology in 1996, and his PhD from Xidian University in 2005. Now, he is the vice president of $\mathrm{Xi}^{\prime}$ an Institute of Space Radio Technology. His main research interest is data transmission and processing.

Zhimei Yang received her BSc degree from Xidian Institute in 2007, and her MSc degree in 2011 from Xi'an Institute of Space Radio Technology. Currently, she is an engineer at Xi'an Institute of Space Radio Technology. Her main research interest is navigation signal processing.

Chenchen Lin received her BSc degree from Harbin Institute of Technology in 2008, and her MSc degree from Harbin Institute of Technology in 2011. Currently, she is a PhD candidate at Xi'an Institute of Space Radio Technology. Her main research interest is moving target detection. 